\title{
Transcranial direct current stimulation: five important issues we aren't discussing (but probably should be)
}

\author{
Jared C. Horvath *, Olivia Carter and Jason D. Forte
}

Psychological Sciences, University of Melbourne, Melbourne, VIC, Australia

\section{Edited by:}

Mikhail Lebedev, Duke University, USA

\section{Reviewed by:}

Carmelo M. Vicario, University of Queensland, Australia

Nick J. Davis, Swansea University, UK

\section{${ }^{*}$ Correspondence:}

Jared C. Horvath, Psychological

Sciences, University of Melbourne,

Redmond Barry Bldg.613,

Melbourne, VIC 3010, Australia

e-mail: jch155@mail.harvard.edu
Transcranial Direct Current Stimulation (tDCS) is a neuromodulatory device often publicized for its ability to enhance cognitive and behavioral performance. These enhancement claims, however, are predicated upon electrophysiological evidence and descriptions which are far from conclusive. In fact, a review of the literature reveals a number of important experimental and technical issues inherent with this device that are simply not being discussed in any meaningful manner. In this paper, we will consider five of these topics. The first, inter-subject variability, explores the extensive between- and within-group differences found within the tDCS literature and highlights the need to properly examine stimulatory response at the individual level. The second, intra-subject reliability, reviews the lack of data concerning tDCS response reliability over time and emphasizes the importance of this knowledge for appropriate stimulatory application. The third, sham stimulation and blinding, draws attention to the importance (yet relative lack) of proper control and blinding practices in the tDCS literature. The fourth, motor and cognitive interference, highlights the often overlooked body of research that suggests typical behaviors and cognitions undertaken during or following tDCS can impair or abolish the effects of stimulation. Finally, the fifth, electric current influences, underscores several largely ignored variables (such as hair thickness and electrode attachments methods) influential to tDCS electric current density and flow. Through this paper, we hope to increase awareness and start an ongoing dialog of these important issues which speak to the efficacy, reliability, and mechanistic foundations of tDCS.

Keywords: transcranial direct current stimulation (tDCS), variability, reliability, efficacy, mechanisms of action

\section{INTRODUCTION}

Transcranial direct current stimulation (tDCS) is currently being promoted as a cheap and effective tool to enhance cognitive and behavioral function. A recent surge of public interest in this device (evidenced by several tDCS devices appearing on the public market) has doubtless been driven by the belief that these enhancement claims are robust, reliable, and well elucidated. However, the research exploring the efficacy of tDCS is far from conclusive.

It's commonly assumed that tDCS shifts the resting membrane potential and synaptic strength of neurons in a predictable and consistent manner. More specifically, hypo-polarization of neurons under the anodal electrode is believed to increase the likelihood of their firing, whilst hyper-polarization of neurons under the cathodal electrode is believed to decrease the likelihood of their firing [for an in depth mechanistic overview, see, Stagg and Nitsche (2011)]. Similar to efficacy, however, a close inspection of the literature reveals short-comings of the anode excite/cathode inhibit model.

In response to efficacy and mechanistic uncertainties, many practitioners focus on the manipulation of three adjustable tDCS parameters: current density, electrode position, and stimulation duration. Whereas these three variables certainly play a large role in tDCS outcomes, there are a number of equally important issues relevant to both efficacy and mechanism that simply are not being discussed in any meaningful manner.

In this paper, we will explore five notable indicators and/or sources of inconsistency associated with the use of tDCS in the current literature: inter-subject variability, intra-subject reliability, lack of effective sham and blinding protocols, motor and $\operatorname{cog}$ nitive interference, and electric current influences. Throughout this piece, we will draw examples solely from studies which explore the effects of tDCS over the motor cortex on MEP amplitude in healthy populations. We have chosen to do this for two reasons: first, MEP amplitude modulation is easily the most explored and reliably demonstrated outcome measure in the tDCS in the literature. Second, as the majority of neurophysiologic, clinical, and behavioral claims cite this work as mechanistically foundational, any issues apparent in this literature will necessarily be applicable to and concern any other outcome measure utilized.

\section{INTER-SUBJECT VARIABILITY}

tDCS must demonstrate similar (or comparable) effects across a range of people before it can be meaningfully applied in healthy and/or clinical populations. However, a survey of the literature reveals extensive between- and within-group variation suggestive of an inconsistent effect between individuals. 
As an example of large between-group variation, Fricke et al. (2011) recently reported data from two different groups that underwent an identical stimulation protocol $\left(0.0286 \mathrm{~mA} / \mathrm{cm}^{2}\right.$ current density; anode M1/cathode contralateral orbit montage; 5 min duration). Whereas one group demonstrated an average MEP amplitude enhancement of $93.2 \%$ in the $5 \mathrm{~min}$ following tDCS, the second group demonstrated an average MEP amplitude enhancement of only 9.2\%: a between-group difference of $913 \%$. Similarly, in two different studies from 2004 using identical stimulation protocols $\left(0.0286 \mathrm{~mA} / \mathrm{cm}^{2}\right.$ current density; cathode M1/anode contralateral orbit montage; 9 min duration), Nitsche et al. reported 30 min group MEP amplitude inhibitions of 42.9\% (Nitsche et al., 2004a) and 20.0\% (Nitsche et al., 2004b): a difference of $110 \%$. Even more variable, these researchers has reported group MEP amplitude enhancements following identical stimulation protocols $\left(0.0286 \mathrm{~mA} / \mathrm{cm}^{2}\right.$ current density; anode $\mathrm{M} 1 /$ cathode contralateral orbit montage; $13 \mathrm{~min}$ duration) ranging from $54.4 \%$ (Nitsche et al., 2003a) to $19.3 \%$ (Nitsche et al., 2009); a difference of $184 \%$.

Specific examples of within-group variability (beyond common deviation and/or error measures) are harder to come by as very few studies include individual data with their reports. However, of the few that have, the results are illuminating. For instance, following $9 \mathrm{~min}$ of anodal stimulation $\left(0.0286 \mathrm{~mA} / \mathrm{cm}^{2}\right.$ current density; M1/contralateral orbit montage), Nitsche and Paulus (2001) reported one subject who demonstrated an incredible 295\% increase in MEP amplitude and a second who demonstrated a weak $5 \%$ increase. More recently, following $20 \mathrm{~min}$ of anodal stimulation $\left(0.06 \mathrm{~mA} / \mathrm{cm}^{2}\right.$ current density; M1/Contralateral orbit montage), Tremblay et al. (2013) reported one subject who demonstrated a $251 \%$ increase in MEP amplitude and a second who demonstrated a $41 \%$ decrease (see also, Roche et al., 2011).

One potential explanation for this extreme between- and within-group variability is the difficulty in properly and reliably targeting TMS pulses during lengthy protocols (Herwig et al., 2001; Sparing et al., 2008; Ahdab et al., 2010). Although modern MRI guided neuronavigation systems can be used to ensure accurate coil positioning across time, many tDCS studies have not utilized (or do not report utilizing) these systems. As such, it is possible subtle variation in coil placement and orientation with time may influence response variation.

A second potential explanation for this extreme betweenand within-group variability is that tDCS generates differential response at the individual level which is masked by group averaging. An individual's unique neurophysiology, anatomy, and psychology may influence his/her response to tDCS. In fact, recent modeling work suggests parameters such as skull thickness, subcutaneous fat levels, cerebrospinal fluid density, and cortical surface topography can greatly influence current flow and density patterns during stimulation (Datta et al., 2012; Truong et al., 2013). As such, elucidation of individual and environmental influences on tDCS is necessary and may only be possible by looking at response characteristics (and related correlative factors) at the individual level.

\section{INTRA-SUBJECT RELIABILITY}

Beyond individual response patterns, it must be demonstrated that people respond in a similar and predictable manner to repeated sessions of tDCS before this tool can be meaningfully applied. Unfortunately, to our knowledge, response reliability at the level of the individual has not been explored (or, at least, reported) in the literature to date.

Of the (only) four studies which have explored group effects of tDCS on MEP amplitude in healthy populations across multiple days, two suggest response patterns may be reliable and replicable. Alonzo et al. (2012) explored the effects of anodal stimulation $\left(0.0571 \mathrm{~mA} / \mathrm{cm}^{2}\right.$ current density; M1/contralateral orbit montage; 20 min duration) on MEP amplitude over the course of 5 days (Monday-Friday). Although these researchers reported variable baseline levels across the week, the ratio of pre- to poststimulation group average MEP amplitudes did not significantly change from day-to-day. Using a similar protocol, Gálvez et al. (2013) reported similar findings: namely, whereas baseline levels changed throughout the week, the group averaged after-effects of daily stimulation did not significantly vary across 5 days.

Interestingly, the remaining two studies to explore group effects of tDCS on MEP amplitude in healthy populations across multiple days suggest response patterns may be unreliable and unpredictable. Monte-Silva et al. have twice looked at the effects of two sessions of tDCS on MEP amplitude with a $24 \mathrm{~h}$ block between sessions (Monte-Silva et al., 2010, 2012). In the first study $\left(0.0286 \mathrm{~mA} / \mathrm{cm}^{2}\right.$ current density; cathodal M1/anodal contralateral orbit montage; $9 \mathrm{~min}$ duration), these researchers reported significantly reduced modulation of MEP amplitude following the second session of stimulation. More concerning, in the second study $\left(0.0286 \mathrm{~mA} / \mathrm{cm}^{2}\right.$ current density; anodal M1/cathodal contralateral orbit montage; 13 min duration) these researchers reported not only a significant reduction in MEP amplitude modulation following the second session of stimulation, but also a reverse in modulation direction (inhibition rather than excitation following anodal stimulation) and unpredictable timing effects.

Considerably more data investigating effects across time is required before concluding $\mathrm{tDCS}$ is a reliable device. As individual response reliability is explored, however, it will be important to remember that intra-subject variability may not, in itself, suggest tDCS is unreliable. It is likely that circadian, metabolic, and hormonal cycles will differentially impact response. In fact, several researchers have already shown that stages of the menstrual cycle and cortisol levels impact plastic response to varied TMS protocols (Smith et al., 1999; Inghilleri et al., 2004; Sale et al., 2008, 2010). In addition, proper and reliable TMS coil positioning during lengthy protocols may also impact response variability (see above). With this in mind, it will certainly be informative to identify the factors that might influence unique tDCS response and whether these factor, themselves, modulate response in a reliable and predictable manner.

\section{SHAM STIMULATION AND BLINDING}

If the dichometric anode excite/cathode inhibit mechanism of tDCS is valid, then comparing the polarities to each other makes determining the true effect of each extremely difficult (as one can never be certain the exact contribution of each polarity 
to the overall difference). Although practitioners aware of this comparative shortcoming extol the use of various control stimulation procedures (such as sham or off-target active stimulation), these procedures have not always proven effective or reliable across varied tDCS protocols (Ambrus et al., 2012; Brunoni et al., 2013; Davis et al., 2013; Palm et al., 2013). In addition, not nearly as many researchers have utilized control conditions as one might expect. In fact, of the 80 studies published to date exploring the effect of $0.0286 \mathrm{~mA} / \mathrm{cm}^{2}$ tDCS current density with an M1/Orbit electrode placement on MEP modulation (the most utilized protocol in the literature), only 10 have compared results to a control condition (Table 1). This means $87.5 \%$ of the studies examining the foundational claim upon which the modern tDCS field is built have not utilized a proper control condition.

Comparing each polarity to its own baseline level (rather than the opposing polarity) does little to address the underlying issues inherent with sham-less protocols. It is commonly acknowledged that MEP amplitude is naturally an extremely variable measure (in fact, Valls-Sole recently pointed out, "The amplitude of MEP to single pulse TMS is not usually employed as a measure of functional relevance because of its large variability and dependence on many technical factors"; p. 9, in press). As such, it can be assumed there will always be some shift away from baseline levels, regardless of intervention (or lack thereof). Accordingly, the utilization of a control condition to differentiate between natural fluctuation and tDCS engendered effects is imperative.

As O'Connell et al. (2012) recently pointed out, tDCS blinding (especially when sham stimulation is being utilized) is of utmost importance yet incredibly difficult to achieve. In fact, these authors reported that, during stimulation using a high current density $\left(0.0571 \mathrm{~mA} / \mathrm{cm}^{2}\right)$, neither the practitioner nor participant was effectively blinded. Beyond this, observable vasodilation (typically over the right orbit) makes practitioner blinding difficult at any current density (see, Palm et al., 2013). Finally, clear sensorial differences between active and sham stimulation (primarily reported as itching, tingling, and/or burning) make blinding participants who undergo multiple conditions difficult at any current density (Davis et al., 2013). As with any scientific study, ineffective blinding may lead to a number of undesirable confounds, including expectation effects, on-the-fly protocol adjustments, and reporting/assessment biases.

In order to elucidate the effects of varied tDCS paradigms, it is essential to continue to amend current and create novel, more effective control conditions. In addition, until such time as more reliable control protocols are developed, it may be beneficial to test for and report blinding procedures and efficacy (or lack-thereof).

\section{MOTOR AND COGNITIVE INTERFERENCE}

Several lines of research suggest that any active motor and/or cognitive activity undertaken during or following tDCS can negatively interfere with or altogether abolish the effects of stimulation. The failure of many practitioners to take account of and further characterize this evidence is concerning.
Quartarone et al. (2004) were the first to report evidence of this interference effect. This group explored MEP amplitude modulation following 5 min of tDCS during motor imagery $\left(0.0286 \mathrm{~mA} / \mathrm{cm}^{2}\right.$ current density; M1/contralateral orbit montage). Whereas imagery (undertaken following stimulation) appeared to prolong the effects of cathodal stimulation, it abolished the effects of anodal stimulation. Despite this important finding (that the act of thinking about motor movement could potentially eliminate tDCS efficacy), this paper went largely ignored and is rarely cited.

Several additional studies have confirmed this interference effect. For instance, Antal et al. (2007) reported that a cognitive task (a combined mathematics, language, geography, and history questionnaire) undertaken during stimulation abolished the effects of both anodal and cathodal stimulation on MEP amplitude modulation $\left(0.0286 \mathrm{~mA} / \mathrm{cm}^{2}\right.$ current density; $\mathrm{M} 1 /$ contralateral orbit montage; 10 min duration). In addition, a simple motor task (pushing around a ball) undertaken during stimulation led to an equivalent decrease in MEP amplitude following both anodal and cathodal stimulation. This equivalent drop suggests the motor activity (perhaps due to fatigue of the target muscle) abolished the effect of stimulation as well. More recently, Miyaguchi et al. (2013) reported that anodal tDCS $\left(0.0571 \mathrm{~mA} / \mathrm{cm}^{2}\right.$ current density; bilateral M1 montage; 10 min duration) delivered with a concurrent non-exhaustive active or passive motor task (self initiated or machine initiated finger abduction-adduction) led to an equivalent MEP amplitude reduction as did undertaking the active motor task alone (without stimulation). Again, this suggests the motor task abolished the effect of stimulation (see also, Thirugnanasambandam et al., 2011).

Secondary evidence for an interference effect can be seen in the often reported diminished tDCS MEP amplitude modulation in voluntarily contracted muscles (common in non-hand targets) compared to resting muscles. For instance, in the $40 \mathrm{~min}$ following cathodal stimulation $\left(0.0286 \mathrm{~mA} / \mathrm{cm}^{2}\right.$ current density; M1/contralateral orbit montage; 15 min duration), Bradnam et al. (2010) reported an average compound MEP decrease of $15.3 \%$ in the right infraspinitus (shoulder) when the muscle was at rest, and an increase of $1.3 \%$ when the muscle was active. Similarly, in the $60 \mathrm{~min}$ following anodal stimulation $\left(0.0571 \mathrm{~mA} / \mathrm{cm}^{2}\right.$ density; M1/contralateral orbit montage; $10 \mathrm{~min}$ duration), Jeffery et al. (2007) reported an average MEP amplitude increase of $34.8 \%$ in the right tibialis anterior (leg) when the muscle was at rest, and an increase of only $25 \%$ when the muscle was activated. Again, these results suggest that motor activity undertaken immediately following stimulation can significantly reduce or eliminate the modulatory effects of tDCS.

These findings suggest that relatively simple and difficult to control for thoughts and/or behaviors may eliminate tDCS efficacy. Clinically, tDCS is often used as an adjunct to physical rehabilitation following stroke (for review, Johansson, 2011). If the aforementioned studies are correct, combining tDCS with motor training may eliminate any desired tDCS effect. This holds true for healthy populations as well. Oftentimes, during long-duration, off-line stimulatory protocols, participants are instructed to simply relax during tDCS. This relaxation can take 
Table 1 | Studies exploring the effects of $0.0286 \mathrm{~mA} / \mathrm{cm}^{2}$ current density, M1/Contralateral Orbit tDCS montage on TMS elicited MEP amplitude of intrinsic hand muscles at rest in healthy participants.

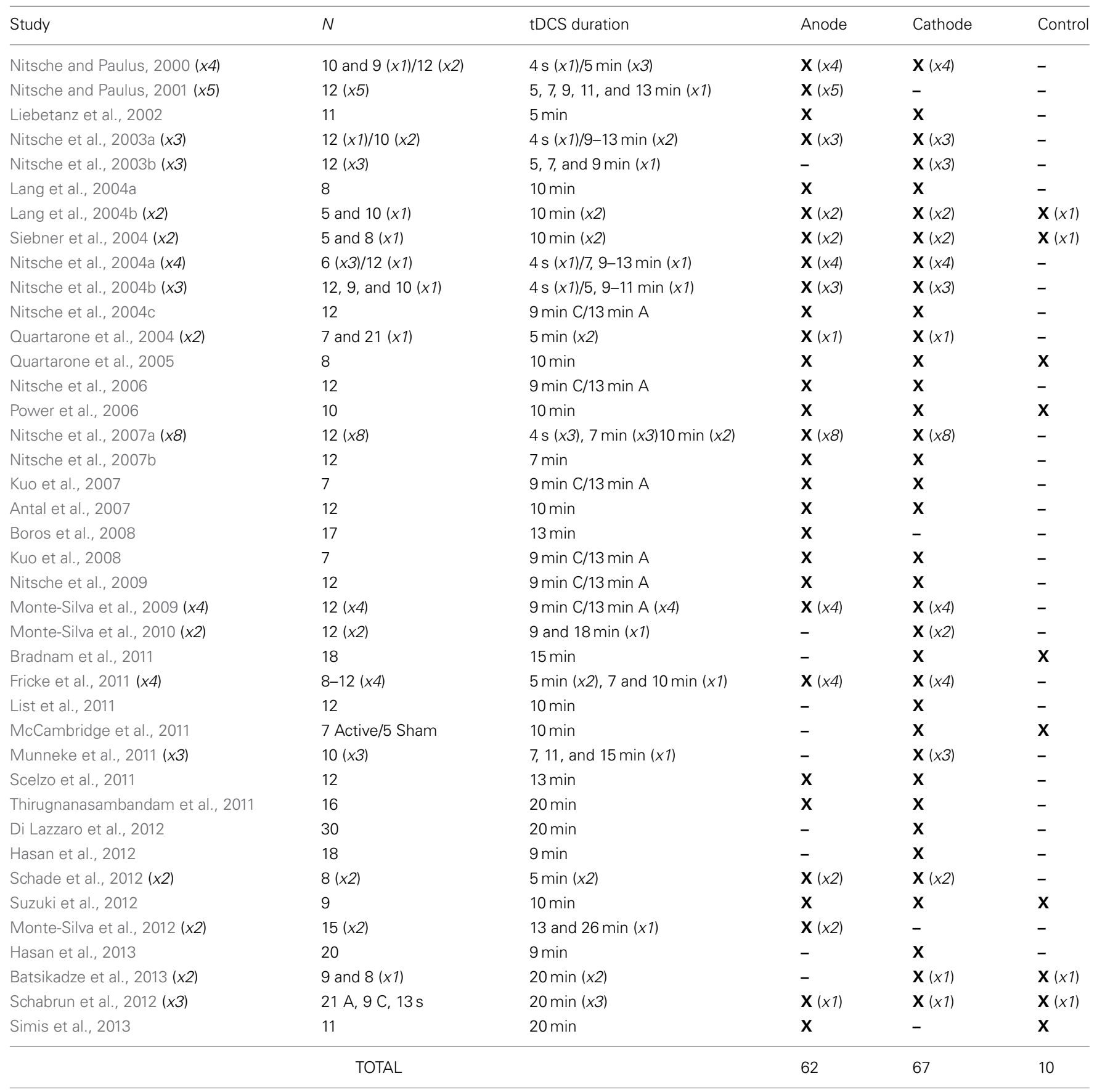

A sham condition from Quartarone et al. (2004) was excluded due to reporting MEP modulation during motor imagery only (not at rest). One study from Monte-Silva et al., 2009 was excluded due to presenting replication data. A, Anode; C, Cathode; S, Sham.

Studies include the placebo condition/s in any drug or device interaction studies.

the form of reading, texting, surfing the internet, doing homework, etc. Is it possible these seemingly innocuous activities are enough to negate or otherwise interfere with the effects of tDCS as well? Additionally, in experiments utilizing MRI to explore the effects of $\mathrm{tDCS}$, stimulation is often given outside the scanning room (although several tDCS devices are now MR compatible). During these protocols, participants must walk back to the scanning room and re-enter the scanner following stimulation: a series of non-trivial motor actions which may, again, interfere with or abolish any tDCS effects. 
If it's possible the effects of tDCS are too weak to manifest during typical human behavior, this is important to determine before more effort and funding are expended utilizing inappropriate protocols. Until such a time as this issue is clearly resolved, it is important practitioners minimize motor and cognitive activity during and immediately following tDCS and during any proceeding procedure (including TMS and/ or MRI).

\section{ELECTRIC CURRENT INFLUENCES}

There are a number of variables which may influence current density and flow to a great extent that have simply not been discussed in the literature to date. Although, as noted above, countless papers have discussed optimal electrode positioning, current density, and stimulation duration for specific outcomes (for discussion, Paulus, 2011), these discussions never seem to evolve past these three parameters.

One variable which may impact current density and flow (but which has yet to be discussed in the literature) is hair thickness. Simply put: hair is not a conductor-it is an insulator. Measurements suggest dry hair $\left(<7 \% 25 \% \mathrm{H}_{2} \mathrm{O}\right.$ content $)$ has a resistivity of approximately $3 \times 10^{12} \Omega / \mathrm{cm}$ whilst wet hair $(25 \%$ $\mathrm{H}_{2} \mathrm{O}$ content) has a resistivity of approximately $6 \times 10^{6} \Omega / \mathrm{cm}$ (Feughelman, 1997). To put that into perspective, skin (the contact surface in many tDCS modeling studies) has a resistivity of approximately $2.15 \times 10^{-2} \Omega / \mathrm{cm}$ (see, Miranda et al., 2006; Wagner et al., 2007: $\Omega=$ Ohm: Note-lower resistivity values equate to higher conductance).

To combat this, practitioners often utilize large amounts of saline to saturate dense hair. Unfortunately, saline spread or dripping at the level of the scalp can guide current flow in undesirable and unpredictable directions. This fact can be easily demonstrated. First, place two saline soaked tDCS sponge electrodes on an easily accessible area of skin (such as the forearms or quadriceps). Next, place a piece of thick, non-conductive plastic under one of the electrodes to ensure no contact is made between the sponge and the skin. Under this set-up, you should be unable to complete the electric circuit. Now, cut a small hole in the plastic barrier (exposing the skin underneath), fill the small hole with saline, and run a continuous stream of saline between the hole and the sponge atop the plastic. Under this new set-up, you should be able to complete the electric circuit quite easily, regardless of how far away from the sponge you have made the small hole (if you are having trouble running a stream of saline between the electrode and the hole, you can substitute a thicker conductive gel: Figure 1).

This demonstration reveals that, even when there is no direct electrode/scalp contact (as may occur in participants with thick hair), excess saline can be used to bridge the tDCS current. However, when this is done, the precise location of the electric current entrance and/or exit points on the scalp will be largely unknown and unpredictable. In addition, when the electric current follows saline to the scalp, the current density also becomes largely unknown and unpredictable (as the number and size of contact points at the scalp becomes uncertain).

Sweat is a second often-ignored variable that may impact electric current dynamics. Because sweat increases skin conductivity

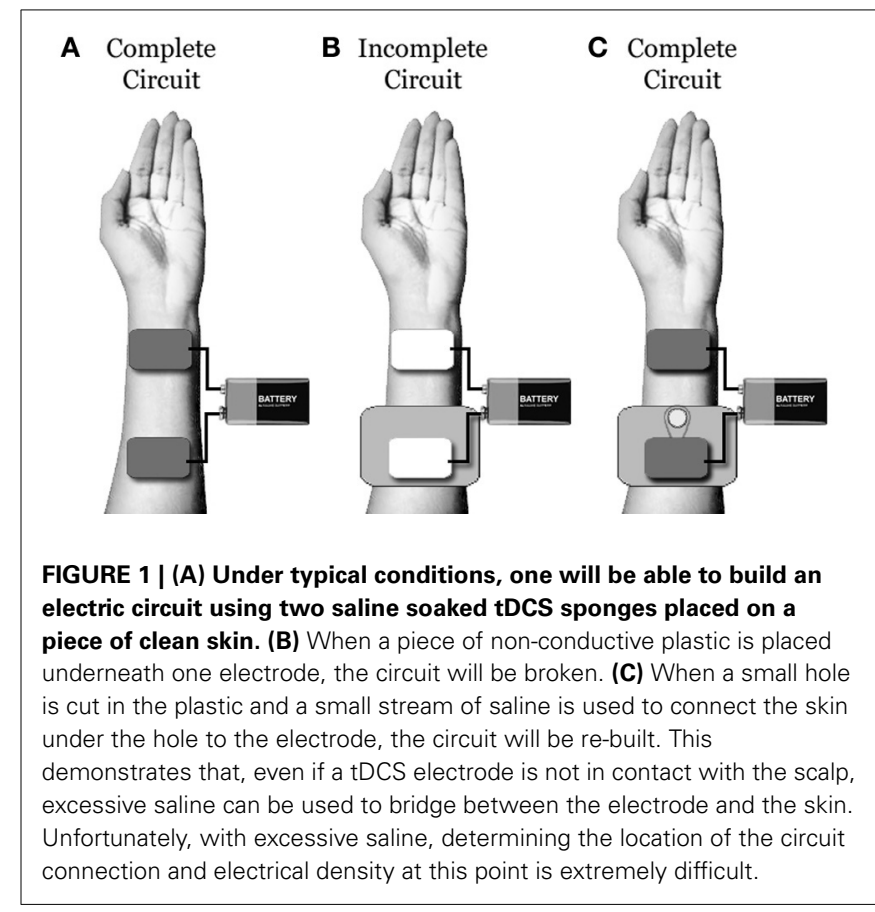

(for review, Dawson et al., 2001), the amount of sweat on a participants scalp may influence current flow in important ways. It's possible that as salts, oils, and electrolytes accumulate in pores on the scalp, the skin will generate enough conductivity to ensure little or no current enters the cortex. However, aside from washing each subject's hair and ensuring a temperature controlled testing environment, how can this be accounted and/or controlled?

Finally, the means by which electrodes are held in place at the scalp may also influence electric current dynamics. For instance, several contemporary tDCS sponge electrodes include plastic rings at the corners (presumably anchor the electrode in place). Unfortunately, unless specifically manufactured, most plastics are non-conductive. Whether or not the plastic used in these electrodes has been produced to conduct electricity is uncertain, although vasodilation patterns following stimulation with these electrodes (which typically reveals no dilatory response underneath the plastic rings themselves) suggests they are not. This may impact current density and flow in unpredictable and uncontrollable ways. In addition, many practitioners hold sponge electrodes in place using rubber straps which are narrower than the electrodes themselves. With these straps, centralized pressure can cause the periphery of the electrodes to "flare" upwards reducing contact area (and, by extension, increasing current density). Given the apparent variability seen within and between individuals (outlined above), it is important to properly consider the influence these (and other) factors may be having on response characteristics.

\section{CONCLUSION}

Recently, several practitioners have noted concerns about modern tDCS conceptions and mechanistic models (Bikson, 2013; Paulus et al., 2013). In addition, a number of studies have also begun to explore response variation in response to adjustments 
in current density, electrode position, and/or stimulation duration (Im et al., 2008; Bikson et al., 2010; Bastani and Jaberzadeh, 2013a,b). Although doubtless important, this work does not address the larger foundational issues raised in this paper.

Although we have chosen to focus on tDCS, many of the issues examined in this paper are applicable to other non-invasive modulatory tools; such as transcranial alternating current stimulation (tACS) and transcranial random noise stimulation (tRNS). These devices are often modified tDCS devices and the protocols utilized by each are often modeled after modern tDCS protocols. Because of this, although there is not enough data in the literature to confidently discuss response variability and reliability, issues of blinding, interference, and electric current influences are highly relevant to these novel tools.

If the field of tDCS is to avoid becoming a footnote in the annals of neuroscientific research, it is time to collectively acknowledge there are shortcomings in our current understanding of this device, its functional parameters, its general efficacy, and its reliability. Rather than seeing the aforementioned issues as a detriment to the field, we should use them to guide future research and exploration. For instance, acknowledging variability can encourage us to explore individual response patterns (and correlate these with related secondary measures to tease-out possible state-dependency effects). Acknowledging the lack of effective sham and blinding techniques can encourage us to develop better more effective devices. Acknowledging the interference effect of motor and/or cognitive activity can inspire us to devise more comprehensive protocols. It is hoped that increased awareness and open discussion of these important issues will lead to a more rigorous and accurate foundation upon which tDCS can be developed into the future.

\section{REFERENCES}

Ahdab, R., Ayache, S. S., Brugieres, P., Goujon, C., and Lefaucheur, J. P. (2010). Comparison of "standard" and "navigated" procedures of TMS coil positioning over motor, premotor and prefrontal targets in patients with chronic pain and depression. Clin. Neurophysiol. 40, 27-36. doi: 10.1016/j.neucli.2010.01.001

Alonzo, A., Brassil, J., Taylor, J. L., Martin, D., and Loo, C. K. (2012). Daily transcranial direct current stimulation (tDCS) leads to greater increases in cortical excitability than second daily transcranial direct current stimulation. Brain Stimul. 5, 208-213. doi: 10.1016/j.brs.2011.04.006

Ambrus, G. G., Al-Moyed, H., Chaieb, L., Sarp, L., Antal, A., and Paulus, W. (2012). The fade-in-short stimulation-fade out approach to sham tDCS-reliable at $1 \mathrm{~mA}$ for naive and experienced subjects, but not investigators. Brain Stimul. 5, 499-504. doi: 10.1016/j.brs.2011.12.001

Antal, A., Terney, D., Poreisz, C., and Paulus, W. (2007). Towards unravelling task-related modulations of neuroplastic changes induced in the human motor cortex. Eur. J. Neurosci. 26, 2687-2691. doi: 10.1111/j.1460-9568.2007.05896.x

Bastani, A., and Jaberzadeh, S. (2013a). a-tDCS differential modulation of corticospinal excitability: the effects of electrode size. Brain Stimul. 6, 932-937. doi: 10.1016/j.brs.2013.04.005

Bastani, A., and Jaberzadeh, S. (2013b). Differential modulation of corticospinal excitability by different current densities of anodal transcranial direct current stimulation. PLoS ONE 8:e72254. doi: 10.1371/journal.pone.0072254

Batsikadze, G., Moliadze, V., Paulus, W., Kuo, M. F., and Nitsche, M. A. (2013). Partially non-linear stimulation intensity-dependent effects of direct current stimulation on motor cortex excitability in humans. J. Physiol. 591, 1987-2000. doi: 10.1113/jphysiol.2012.249730

Bikson, M. (2013). Origins of specificity during tDCS: anatomical, activityselective, and input-bias mechanisms. Front. Hum. Neurosci. 7:688. doi: 10.3389/fnhum.2013.00688
Bikson, M., Datta, A., Rahman, A., and Scaturro, J. (2010). Electrode montages for tDCS and weak transcranial electrical stimulation: role of "return" electrode's position and size. Clin. Neurophysiol. 121, 1976. doi: 10.1016/j.clinph.2010.05.020

Boros, K., Poreisz, C., Münchau, A., Paulus, W., and Nitsche, M. A. (2008). Premotor transcranial direct current stimulation (tDCS) affects primary motor excitability in humans. Eur. J. Neurosci. 27, 1292-1300. doi: 10.1111/j.14609568.2008.06090.x

Bradnam, L. V., Stinear, C. M., and Byblow, W. D. (2011). Cathodal transcranial direct current stimulation suppresses ipsilateral projections to presumed propriospinal neurons of the proximal upper limb. J. Neurophysiol. 105, 2582-2589. doi: 10.1152/jn.01084.2010

Bradnam, L. V., Stinear, C. M., Lewis, G. N., and Byblow, W. D. (2010). Taskdependent modulation of inputs to proximal upper limb following transcranial direct current stimulation of primary motor cortex. J. Neurophysiol. 103, 2382-2389. doi: 10.1152/jn.01046.2009

Brunoni, A. R., Schestatsky, P., Lotufo, P. A., Benseñor, I. M., and Fregni, F. (2013). Comparison of blinding effectiveness between sham tDCS and placebo sertraline in a 6-week major depression randomized clinical trial. Clin. Neurophysiol. doi: 10.1016/j.clinph.2013.07.020. [Epub ahead of print].

Datta, A., Truong, D., Minhas, P., Parra, L. C., and Bikson, M. (2012). Interindividual variation during transcranial direct current stimulation and normalization of dose using MRI-derived computational models. Front. Psychiatry 3:91. doi: 10.3389/fpsyt.2012.00091

Davis, N. J., Gold, E., Pascual-Leone, A., and Bracewell, R. M. (2013). Challenges of proper placebo control for non-invasive brain stimulation in clinical and experimental applications. Eur. J. Neurosci. 38, 2973-2977. doi: 10.1111/ejn.12307

Dawson, M. E., Schell, A. M., and Filion, D. L. (2001). “The electrodermal system," in Handbook of Psycholphysiology, eds J. T. Cacioppo, L. G. Tassinary, and G. G. Berntson (Cambridge, UK: Cambridge University Press), 159-181.

Di Lazzaro, V., Manganelli, F., Dileone, M., Notturno, F., Esposito, M., Capasso, M., et al. (2012). The effects of prolonged cathodal direct current stimulation on the excitatory and inhibitory circuits of the ipsilateral and contralateral motor cortex. J. Neural Transm. 119, 1499-1506. doi: 10.1007/s00702-0120845-4

Feughelman, M. (1997). Mechanical Properties and Structure of Alpha-Keratin Fibres: Wool, Human Hair, and Related Fibres. Sydney, NSW: University of New South Wales Press.

Fricke, K., Seeber, A. A., Thirugnanasambandam, N., Paulus, W., Nitsche, M. A., and Rothwell, J. C. (2011). Time course of the induction of homeostatic plasticity generated by repeated transcranial direct current stimulation of the human motor cortex. J. Neurophysiol. 105, 1141-1149. doi: 10.1152/jn. 00608.2009

Gálvez, V., Alonzo, A., Martin, D., and Loo, C. K. (2013). Transcranial direct current stimulation treatment protocols: should stimulus intensity be constant or incremental over multiple sessions. Int. J. Neuropsychopharmacol. 16, 13-21. doi: 10.1017/S1461145712000041

Hasan, A., Aborowa, R., Nitsche, M. A., Marshall, L., Schmitt, A., Gruber, O., et al. (2012). Abnormal bihemispheric responses in schizophrenia patients following cathodal transcranial direct stimulation. Eur. Arch. Psychiatry Clin. Neurosci. 262, 415-423. doi: 10.1007/s00406-012-0298-7

Hasan, A., Misewitsch, K., Nitsche, M. A., Gruber, O., Padberg, F., Falkai, P., et al. (2013). Impaired motor cortex responses in non-psychotic first-degree relatives of schizophrenia patients: a cathodal tDCS pilot study. Brain Stimul. 6, 821-829. doi: 10.1016/j.brs.2013.03.001

Herwig, U., Padberg, F., Unger, J., Spitzer, M., and Schönfeldt-Lecuona, C. (2001). Transcranial magnetic stimulation in therapy studies: examination of the reliability of "standard" coil positioning by neuronavigation. Biol. Psychiatry 50, 58-61. doi: 10.1016/S0006-3223(01)01153-2

Im, C. H., Jung, H. H., Choi, J. D., Lee, S. Y., and Jung, K. Y. (2008). Determination of optimal electrode positions for transcranial direct current stimulation (tDCS). Phys. Med. Biol. 53, N219. doi: 10.1088/0031-9155/53/11/N03

Inghilleri, M., Conte, A., Curra, A., Frasca, V., Lorenzano, C., and Berardelli, A. (2004). Ovarian hormones and cortical excitability. An rTMS study in humans. Clin. Neurophysiol. 115, 1063-1068. doi: 10.1016/j.clinph.2003. 12.003

Jeffery, D. T., Norton, J. A., Roy, F. D., and Gorassini, M. A. (2007). Effects of transcranial direct current stimulation on the excitability of the leg motor cortex. Exp. Brain Res. 182, 281-287. doi: 10.1007/s00221-007-1093-y 
Johansson, B. B. (2011). Current trends in stroke rehabilitation. A review with focus on brain plasticity. Acta Neurol. Scand. 123, 147-159. doi: 10.1111/j.16000404.2010.01417.x

Kuo, M. F., Grosch, J., Fregni, F., Paulus, W., and Nitsche, M. A. (2007). Focusing effect of acetylcholine on neuroplasticity in the human motor cortex. J. Neurosci. 27, 14442-14447. doi: 10.1523/JNEUROSCI.4104-07.2007

Kuo, M. F., Paulus, W., and Nitsche, M. A. (2008). Boosting focally-induced brain plasticity by dopamine. Cereb. Cortex 18, 648-651. doi: 10.1093/cercor/bhm098

Lang, N., Nitsche, M. A., Paulus, W., Rothwell, J. C., and Lemon, R. N. (2004a). Effects of transcranial direct current stimulation over the human motor cortex on corticospinal and transcallosal excitability. Exp. Brain Res. 156, 439-443. doi: 10.1007/s00221-003-1800-2

Lang, N., Siebner, H. R., Ernst, D., Nitsche, M. A., Paulus, W., Lemon, R. N., et al. (2004b). Preconditioning with transcranial direct current stimulation sensitizes the motor cortex to rapid-rate transcranial magnetic stimulation and controls the direction of after-effects. Biol. Psychiatry 56, 634-639. doi: 10.1016/j.biopsych.2004.07.017

Liebetanz, D., Nitsche, M. A., Tergau, F., and Paulus, W. (2002). Pharmacological approach to the mechanisms of transcranial DC-stimulation-induced after-effects of human motor cortex excitability. Brain 125, 2238-2247. doi: 10.1093/brain/awf238

List, J., Duning, T., Meinzer, M., Kürten, J., Schirmacher, A., Deppe, M., et al. (2011). Enhanced rapid-onset cortical plasticity in CADASIL as a possible mechanism of preserved cognition. Cereb. Cortex 21, 2774-2787. doi: 10.1093/cercor/bhr071

McCambridge, A. B., Bradnam, L. V., Stinear, C. M., and Byblow, W. D. (2011). Cathodal transcranial direct current stimulation of the primary motor cortex improves selective muscle activation in the ipsilateral arm. J. Neurophysiol. 105, 2937-2942. doi: 10.1152/jn.00171.2011

Miranda, P. C., Lomarev, M., and Hallett, M. (2006). Modeling the current distribution during transcranial direct current stimulation. Clin. Neurophysiol. 117, 1623-1629. doi: 10.1016/j.clinph.2006.04.009

Miyaguchi, S., Onishi, H., Kojima, S., Sugawara, K., Tsubaki, A., Kirimoto, H., et al. (2013). Corticomotor excitability induced by anodal transcranial direct current stimulation with and without non-exhaustive movement. Brain Res. 1529, 83-91.

Monte-Silva, K., Kuo, M. F., Hessenthaler, S., Fresnoza, S., Liebetanz, D., Paulus, W., et al. (2012). Induction of late LTP-like plasticity in the human motor cortex by repeated non-invasive brain stimulation. Brain Stimul. 6, 424-432. doi: 10.1016/j.brs.2012.04.011

Monte-Silva, K., Kuo, M. F., Liebetanz, D., Paulus, W., and Nitsche, M. A. (2010). Shaping the optimal repetition interval for cathodal transcranial direct current stimulation (tDCS). J. Neurophysiol. 103, 1735-1740. doi: 10.1152/jn.00924.2009

Monte-Silva, K., Kuo, M. F., Thirugnanasambandam, N., Liebetanz, D., Paulus, W., and Nitsche, M. A. (2009). Dose-dependent inverted U-shaped effect of dopamine (D2-like) receptor activation on focal and nonfocal plasticity in humans. J. Neurosci. 29, 6124-6131. doi: 10.1523/JNEUROSCI.0728-09.2009

Munneke, M. A., Stegeman, D. F., Hengeveld, Y. A., Rongen, J. J., Schelhaas, H. J., and Zwarts, M. J. (2011). Transcranial direct current stimulation does not modulate motor cortex excitability in patients with amyotrophic lateral sclerosis. Muscle Nerve 44, 109-114. doi: 10.1002/mus.22012

Nitsche, M. A., Doemkes, S., Karakoese, T., Antal, A., Liebetanz, D., Lang, N., et al. (2007a). Shaping the effects of transcranial direct current stimulation of the human motor cortex. J. Neurophysiol. 97, 3109-3117. doi: 10.1152/jn.01312.2006

Nitsche, M. A., Roth, A., Kuo, M. F., Fischer, A. K., Liebetanz, D., Lang, N., et al. (2007b). Timing-dependent modulation of associative plasticity by general network excitability in the human motor cortex. J. Neurosci. 27, 3807-3812. doi: 10.1523/JNEUROSCI.5348-06.2007

Nitsche, M. A., Fricke, K., Henschke, U., Schlitterlau, A., Liebetanz, D., Lang, N., et al. (2003a). Pharmacological modulation of cortical excitability shifts induced by transcranial direct current stimulation in humans. J. Physiol. 553, 293-301. doi: 10.1113/jphysiol.2003.049916

Nitsche, M. A., Nitsche, M. S., Klein, C. C., Tergau, F., Rothwell, J. C., and Paulus, W. (2003b). Level of action of cathodal DC polarisation induced inhibition of the human motor cortex. Clin. Neurophysiol. 114, 600-604. doi: 10.1016/S13882457(02)00412-1
Nitsche, M. A., Grundey, J., Liebetanz, D., Lang, N., Tergau, F., and Paulus, W. (2004a). Catecholaminergic consolidation of motor cortical neuroplasticity in humans. Cereb. Cortex 14, 1240-1245. doi: 10.1093/cercor/bhh085

Nitsche, M. A., Liebetanz, D., Schlitterlau, A., Henschke, U., Fricke, K., Frommann, K., et al. (2004b). GABAergic modulation of DC stimulation-induced motor cortex excitability shifts in humans. Eur. J. Neurosci. 19, 2720-2726. doi: 10.1111/j.0953-816X.2004.03398.x

Nitsche, M. A., Jaussi, W., Liebetanz, D., Lang, N., Tergau, F., and Paulus, W. (2004c). Consolidation of human motor cortical neuroplasticity by Dcycloserine. Neuropsychopharmacology 29, 1573. doi: 10.1038/sj.npp.1300517

Nitsche, M. A., Kuo, M. F., Karrasch, R., Wächter, B., Liebetanz, D., and Paulus, W. (2009). Serotonin affects transcranial direct current-induced neuroplasticity in humans. Biol. Psychiatry 66, 503-508. doi: 10.1016/j.biopsych.2009.03.022

Nitsche, M. A., Lampe, C., Antal, A., Liebetanz, D., Lang, N., Tergau, F., et al. (2006). Dopaminergic modulation of long-lasting direct current-induced cortical excitability changes in the human motor cortex. Eur. J. Neurosci. 23, 1651-1657. doi: 10.1111/j.1460-9568.2006.04676.x

Nitsche, M. A., and Paulus, W. (2000). Excitability changes induced in the human motor cortex by weak transcranial direct current stimulation. J. Physiol. 527, 633-639. doi: 10.1111/j.1469-7793.2000.t01-1-00633.x

Nitsche, M. A., and Paulus, W. (2001). Sustained excitability elevations induced by transcranial DC motor cortex stimulation in humans. Neurology 57, 1899-1901. doi: 10.1212/WNL.57.10.1899

O’Connell, N. E., Cossar, J., Marston, L., Wand, B. M., Bunce, D., Moseley, G. L., et al. (2012). Rethinking clinical trials of transcranial direct current stimulation: participant and assessor blinding is inadequate at intensities of $2 \mathrm{~mA}$. PLoS ONE 7:e47514. doi: 10.1371/journal.pone.0047514

Palm, U., Reisinger, E., Keeser, D., Kuo, M. F., Pogarell, O., Leicht, G., et al. (2013). Evaluation of sham transcranial direct current stimulation for randomized, placebo-controlled clinical trials. Brain Stimul. 6, 690-695. doi: 10.1016/j.brs.2013.01.005

Paulus, W. (2011). Transcranial electrical stimulation (tES-tDCS; tRNS, tACS) methods. Neuropsychol. Rehabil. 21, 602-617. doi: 10.1080/09602011.2011.557292

Paulus, W., Peterchev, A. V., and Ridding, M. (2013). "Transcranial electric and magnetic stimulation: technique and paradigms," in Brain Stimulation E-Book: Handbook of Clinical Neurology, Vol. 116, Series eds M. J. Aminoff, F. Boller, and D. F. Swaab (Amsterdam, ND: Elsevier B.V.), 329.

Power, H. A., Norton, J. A., Porter, C. L., Doyle, Z., Hui, I., and Chan, K. M. (2006). Transcranial direct current stimulation of the primary motor cortex affects cortical drive to human musculature as assessed by intermuscular coherence. J. Physiol. 577, 795-803. doi: 10.1113/jphysiol.2006.116939

Quartarone, A., Morgante, F., Bagnato, S., Rizzo, V., Sant'Angelo, A., Aiello, E., et al. (2004). Long lasting effects of transcranial direct current stimulation on motor imagery. Neuroreport 15, 1287-1291. doi: 10.1097/01.wnr.0000127637. $22805.7 \mathrm{c}$

Quartarone, A., Rizzo, V., Bagnato, S., Morgante, F., Sant'Angelo, A., Romano, M., et al. (2005). Homeostatic-like plasticity of the primary motor hand area is impaired in focal hand dystonia. Brain 128, 1943-1950. doi: 10.1093/brain/awh527

Roche, N., Lackmy, A., Achache, V., Bussel, B., and Katz, R. (2011). Effects of anodal transcranial direct current stimulation over the leg motor area on lumbar spinal network excitability in healthy subjects. J. Physiol. 589, 2813-2826. doi: 10.1113/jphysiol.2011.205161

Sale, M. V., Ridding, M. C., and Nordstrom, M. A. (2008). Cortisol inhibits neuroplasticity induction in human motor cortex. J. Neurosci. 28, 8285-8293. doi: 10.1523/JNEUROSCI.1963-08.2008

Sale, M. V., Ridding, M. C., and Nordstrom, M. A. (2010). Circadian modulation off neuroplasticity in humans and potential therapeutic implications. Rev. Neurosci. 21, 55-66. doi: 10.1515/REVNEURO.2010.21.1.55

Scelzo, E., Giannicola, G., Rosa, M., Ciocca, M., Ardolino, G., Cogiamanian, F., et al. (2011). Increased short latency afferent inhibition after anodal transcranial direct current stimulation. Neurosci. Lett. 498, 167-170. doi: 10.1016/j.neulet.2011.05.007

Schabrun, S. M., Chipchase, L. S., Zipf, N., Thickbroom, G. W., and Hodges, P. W. (2012). Interaction between simultaneously applied neuromodulatory interventions in humans. Brain Stimul. 6, 624-630. doi: 10.1016/j.brs.2012. 09.009 
Schade, S., Moliadze, V., Paulus, W., and Antal, A. (2012). Modulating neuronal excitability in the motor cortex with tDCS shows moderate hemispheric asymmetry due to subjects' handedness: a pilot study. Restor. Neurol. Neurosci. 30, 191-198. doi: 10.3233/RNN-2012-110175

Siebner, H. R., Lang, N., Rizzo, V., Nitsche, M. A., Paulus, W., Lemon, R. N., et al. (2004). Preconditioning of low-frequency repetitive transcranial magnetic stimulation with transcranial direct current stimulation: evidence for homeostatic plasticity in the human motor cortex. J. Neurosci. 24, 3379-3385. doi: 10.1523/JNEUROSCI.5316-03.2004

Simis, M., Adeyemo, B. O., Medeiros, L. F., Miraval, F., Gagliardi, R. J., and Fregni, F. (2013). Motor cortex-induced plasticity by noninvasive brain stimulation: a comparison between transcranial direct current stimulation and transcranial magnetic stimulation. Neuroreport 24, 973-975. doi: 10.1097/WNR.0000000000000021

Smith, M. J., Keel, J. C., Greenberg, B. D., Adams, L. F., Schmidt, P. J., Rubinow, D. A., et al. (1999). Menstrual cycle effects on cortical excitability. Neurology 53, 2069-2069. doi: 10.1212/WNL.53.9.2069

Sparing, R., Buelte, D., Meister, I. G., Pauš, T., and Fink, G. R. (2008). Transcranial magnetic stimulation and the challenge of coil placement: a comparison of conventional and stereotaxic neuronavigational strategies. Hum. Brain Mapp. 29, 82-96. doi: 10.1002/hbm.20360

Stagg, C. J., and Nitsche, M. A. (2011). Physiological basis of transcranial direct current stimulation. Neuroscientist 17, 37-53. doi: 10.1177/ 1073858410386614

Suzuki, K., Fujiwara, T., Tanaka, N., Tsuji, T., Masakado, Y., Hase, K., et al. (2012). Comparison of the after-effects of transcranial direct current stimulation over the motor cortex in patients with stroke and healthy volunteers. Int. J. Neurosci. 122, 675-681. doi: 10.3109/00207454.2012.707715

Thirugnanasambandam, N., Sparing, R., Dafotakis, M., Meister, I. G., Paulus, W., Nitsche, M. A., et al. (2011). Isometric contraction interferes with transcranial direct current stimulation (tDCS) induced plasticity-evidence of state-dependent neuromodulation in human motor cortex. Restor. Neurol. Neurosci. 29, 311-320. doi: 10.3233/RNN-2011-0601

Tremblay, S., Beaulé, V., Lepage, J. F., and Théoret, H. (2013). Anodal transcranial direct current stimulation modulates GABAB-related intracortical inhibition in the M1 of healthy individuals. Neuroreport 24, 46-50. doi: 10.1097/WNR.0b013e32835c36b8

Truong, D. Q., Magerowski, G., Blackburn, G. L., Bikson, M., and Alonso-Alonso, M. (2013). Computational modeling of transcranial direct current stimulation (tDCS) in obesity: impact of head fat and dose guidelines. Neuroimage Clin. 2, 759-766. doi: 10.1016/j.nicl.2013.05.011

Wagner, T., Fregni, F., Fecteau, S., Grodzinsky, A., Zahn, M., and Pascual-Leone, A. (2007). Transcranial direct current stimulation: a computer-based human model study. Neuroimage 35, 1113-1124. doi: 10.1016/j.neuroimage.2007.01.027

Conflict of Interest Statement: The authors declare that the research was conducted in the absence of any commercial or financial relationships that could be construed as a potential conflict of interest.

Received: 18 December 2013; accepted: 08 January 2014; published online: 24 January 2014.

Citation: Horvath JC, Carter O and Forte JD (2014) Transcranial direct current stimulation: five important issues we aren't discussing (but probably should be). Front. Syst. Neurosci. 8:2. doi: 10.3389/fnsys.2014.00002

This article was submitted to the journal Frontiers in Systems Neuroscience.

Copyright (c) 2014 Horvath, Carter and Forte. This is an open-access article distributed under the terms of the Creative Commons Attribution License (CC BY). The use, distribution or reproduction in other forums is permitted, provided the original author(s) or licensor are credited and that the original publication in this journal is cited, in accordance with accepted academic practice. No use, distribution or reproduction is permitted which does not comply with these terms. 